\title{
Chronic Stress Prevents Cortico-Accumbens Cue Encoding and Alters Conditioned Approach
}

\author{
Mitchell G. Spring, Aaron Caccamise, Elizabeth A. Panther, Bethany M. Windsor, Karan R. Soni, \\ Jayme R. McReynolds, Daniel S. Wheeler, John R. Mantsch, and Robert A. Wheeler \\ Department of Biomedical Sciences, Marquette University, Milwaukee, Wisconsin 53233
}

Chronic stress impairs the function of multiple brain regions and causes severe hedonic and motivational deficits. One brain region known to be susceptible to these effects is the PFC. Neurons in this region, specifically neuronal projections from the prelimbic region (PL) to the nucleus accumbens core (NAcC), have a significant role in promoting motivated approach. However, little is known about how activity in this pathway changes during associative learning to encode cues that promote approach. Less is known about how activity in this pathway may be altered by stress. In this study, an intersectional fiber photometry approach was used in male Sprague Dawley rats engaged in a Pavlovian autoshaping design to characterize the involvement of the PL-NAcC pathway in the typical acquisition of learned approach (directed at both the predictive cue and the goal), and its potential alteration by stress. Specifically, the hypothesis that neural activity in PL-NAcC would encode a Pavlovian approach cue and that prior exposure to chronic stress would disrupt both the nature of conditioned approach and the encoding of a cue that promotes approach was tested. Results of the study demonstrated that the rapid acquisition of conditioned approach was associated with cue-induced PL-NAcC activity. Prior stress both reduced cue-directed behavior and impaired the associated cortical activity. These findings demonstrate that prior stress diminishes the task-related activity of a brain pathway that regulates approach behavior. In addition, the results support the interpretation that stress disrupts reward processing by altering the incentive value of associated cues.

Key words: approach; learning; motivation; prefrontal; reward; stress

\section{Significance Statement}

Chronic stress causes hedonic and motivational deficits and disrupts the function of the PFC. A specific projection from the prelimbic region of the PFC to the nucleus accumbens core (PL-NAcC) promotes approach behavior and is a strong candidate for contributing to stress-induced disruptions in motivation. However, it is not known how activity in this pathway encodes cues that promote approach, and how this encoding may be altered by stress. Here we show that the rapid acquisition of conditioned approach is associated with cue-induced activity in the PL-NAcC pathway. Prior stress both reduces cue-directed behavior and impairs the associated cortical activity. These findings demonstrate that stress diminishes task-related activity in a brain pathway that regulates approach behavior.

\section{Introduction}

Mood disorders are debilitating, in part, because they involve severe hedonic and motivational deficits. These same symptoms

Received July 17, 2020; revised Feb. 8, 2021; accepted Feb. 10, 2021.

Author contributions: M.G.S., D.S.W., J.R. Mantsch, and R.A.W. designed research; M.G.S., A.C., E.A.P., B.M.W., K.R.S., and J.R. McReynolds performed research; M.G.S., E.A.P., B.M.W., D.S.W., and R.A.W. analyzed data; M.G.S. wrote the first draft of the paper; M.G.S., D.S.W., and R.A.W. wrote the paper; A.C., E.A.P., B.M.W., K.R.S., J.R. McReynolds, D.S.W., J.R. Mantsch, and R.A.W. edited the paper; J.R. McReynolds contributed unpublished reagents/analytic tools.

This work was supported by the Charles E. Kubly Mental Health Research Center and National Institutes of Health DA048280 to R.A.W. and J.R. Mantsch and DA038663 to J.R. Mantsch We thank Talia Lerner, Vaibhav Konanur, and Mitch Roitman for technical assistance.

The authors declare no competing financial interests.

Correspondence should be addressed to Robert A. Wheeler at robert.wheeler@ marquette.edu.

https://doi.org/10.1523/JNEUROSCI.1869-20.2021

Copyright $\odot 2021$ the authors are associated with several types of regressive neuroplasticity induced by chronic stress exposure (Price and Duman, 2020). This close relationship makes stress a useful procedural tool for invasive studies attempting to identify the dysfunctional brain circuits that produce depressive-like symptoms (Willner, 2017). Unfortunately, it can be difficult to disentangle hedonic and motivational deficits that are caused by stress. For example, anhedonia is traditionally defined as the inability to experience pleasure (Ribot, 1896), and it is considered a hallmark of both major depressive disorder and the efficacy of a stress procedure (Drysdale et al., 2017; Willner, 2017; Rizvi et al., 2018). However, anhedonia is not universally observed in major depressive disorder (Thomsen et al., 2015; Rizvi et al., 2018) and is present in a much wider range of pathologies (Insel and Cuthbert, 2015). Adding to this complexity is the fact that tests of compromised hedonic processing are often also sensitive to disruptions in 
motivation, suggesting that behavioral disruptions assumed to be signs of anhedonia may instead be the result of impaired approach motivation (Olney et al., 2018). Focusing on symptoms rather than disorders, then, is critical; and expanding approaches to better characterize these symptoms is critical as well. For this reason, conditioned approach designs may be useful for characterizing the disruptive effects of stress.

Conditioned approach behavior (i.e., approach elicited by a reward-paired cue) is an essential behavior for the navigation of an animal's environment. Conditioning parameters have a significant effect on the nature of this approach. Purely visual or auditory Pavlovian cues promote approach directed toward the site of reward delivery. However, when a physical cue is used in conditioning, as in Pavlovian autoshaping, animals display parameter-dependent variability in the direction of approach (Robinson and Flagel, 2009; Meyer et al., 2012). Some animals display reward-site directed behavior. Others express a remarkable degree of cue interaction, often going well beyond approach behavior, appearing to attempt to "consume" the cue as though it were the reward (Davey and Cleland, 1982). The difference between the two types of learned responses is thought to reflect a difference in reward value that is attached to the physical cue, with the transfer of conditioned incentive leading to vigorous interaction with the otherwise neutral cue (Robinson and Flagel, 2009). The development of conditioned approach has been used to assess the effects of circuit manipulation on hedonic versus incentive valuation processes (e.g., Berridge et al., 2009) and can be impaired by exposure to prolonged stress (Fitzpatrick et al., 2019).

Neurons in the PFC are susceptible to the regressive neuroplasticity caused by stress (Radley et al., 2006; Dias-Ferreira et al., 2009) and are well positioned to guide approach behavior. In particular, neuronal projections from the prelimbic (PL) PFC to the nucleus accumbens core (NAcC) serve as a critical substrate for motivated approach (McFarland et al., 2003; Vialou et al., 2014). Activity in this pathway is causally related to conditioned appetitive responses elicited by a reward-predictive cue (Otis et al., 2017). Although the pathway itself has not been studied extensively in autoshaping designs, cue presentation has been shown to promote glutamatergic signaling in both the PL and NAcC of sign-tracking rats (Batten et al., 2018). Given the sensitivity of the PFC to stress and the involvement of the PL-NAcC pathway in directing motivated approach, this study isolated and characterized its involvement in the acquisition of conditioned approach. Specifically, this study tested the hypothesis that neural activity in PL-NAcC would encode a Pavlovian approach cue, and that prior exposure to chronic stress would disrupt both the nature of conditioned approach and the encoding of a cue that promotes approach.

\section{Materials and Methods \\ Animals}

Adult male Sprague Dawley rats (300-350 g; Harlan Laboratories) were used in all experiments. Animals were individually housed on a reverse 12:12 light-dark cycle in a temperature- and humidity-controlled, Association for Assessment and Accreditation of Laboratory Animal Care-accredited vivarium. All procedures were approved by the Marquette University Institutional Animal Care and Use Committee. All animals were trained in autoshaping $(N=59)$, with half being exposed to chronic variable stress $(n=30)$. A subset of animals received fiber photometry surgery, and those animals with both confirmed fiber placement and GCaMP expression contributed data to both the behavior and photometry analyses (Stress: $n=8$; Control: $n=13$ ). During autoshaping training and for $3 \mathrm{~d}$ prior, animals were fed standard chow (TekLad) once daily to maintain $95 \%$ body weight. Water was available ad libitum for the duration of all experiments.

\section{Chronic variable stress (CVS)}

The CVS regimen was a $14 \mathrm{~d}$ procedure consisting of exposure to 2 of the following stressors per day: forced swim $\left(4^{\circ} \mathrm{C}\right.$ water for $\left.20 \mathrm{~min}\right)$, cold room $\left(4^{\circ} \mathrm{C}, 2 \mathrm{~h}\right.$, alone or in combination with other stressors), novel environment (different novel environments for 1-3 h; including wet bedding in cages, one-half inch of water in cages, or no bedding in cages), motion (cage without bedding, which is placed on an orbital shaker and rotated for $2 \mathrm{~h}$; 1 revolution/s), noise (continuous 60-68 dB noise, e.g., radio static for $1 \mathrm{~h}$ ), open field (alone or in groups in a 1-m-diameter circular brightly illuminated field for $45 \mathrm{~min}$ ), restraint (plastic restraint tubes for $30 \mathrm{~min}$ ), and cage tilt ( $30^{\circ}$ for $6-12 \mathrm{~h}$ with food and water available). For repeating stressors, variables, such as light, temperature, and noise, were varied to maintain novelty. On each day over the $14 \mathrm{~d}$ period, one of the stressors from the battery was presented at $0800 \mathrm{~h}$ and the other stressor was presented at $1700 \mathrm{~h}$. Control rats were handled and weighed daily at the evening time point.

\section{Autoshaping procedure}

Pavlovian autoshaping took place in Plexiglas operant chambers (MED Associates) housed within sound-attenuating boxes (Stanley Vidmar). Two retractable levers flanked a centrally located food cup on one wall of the operant chamber. For animals that did not contribute photometry data, this food cup was recessed; for animals in photometry experiments, this food cup extended into the cage to prevent the optic fiber from interfering with pellet retrieval. This minor chamber adjustment prevented automated photobeam detection of goal approach behavior for the subset of animals that contributed photometric data. For these animals, video analysis provided goal approach behavioral measures. Cue lights were located above each lever. A house light placed on the opposite wall illuminated the chamber.

Daily $1 \mathrm{~h}$ training sessions comprised 50 trials. For 25 trials, the lever and light on one side of the food hopper were extended and illuminated for $10 \mathrm{~s}$, after which a sucrose pellet $(45 \mathrm{mg}$; Bio-Serv) was delivered to the food cup (conditioned stimulus $[\mathrm{CS}]^{+}$trials). In another 25 trials, the lever and light on the other side of the cup were presented in the same manner but were not followed by sucrose delivery ( $\mathrm{CS}^{-}$trials). CS presentations occurred in pseudorandom order such that no more than two trials of a single type occurred sequentially. Random intertrial intervals, with an average duration of $60 \mathrm{~s}$, separated CS trials. During each session, behavioral data (including lever interactions, head entries into the goal box, and pellet consumption) were collected. The food hopper was checked at the beginning and end of each session to verify pellet delivery. On the rare occasion in which pellet delivery was interrupted because of an equipment malfunction, data from that day of training were omitted from analyses.

\section{Behavioral analysis}

Autoshaping acquisition was first characterized by calculating the probability of lever approach. This probability was calculated as the [number of trials of a given type $\left(\mathrm{CS}^{+}\right.$or $\left.\mathrm{CS}^{-}\right)$in which at least one lever contact was made]/[number of trials of corresponding type]. For all animals, lever contacts were recorded automatically on lever deflection. Head entry information was also scored for all animals. Automated photobeam detection of head entries into the goal box was not possible for animals that contributed photometry data. For these animals, video-recording (10 frames/s) of behavior was used to score head entries. A head entry was defined by the animal's nose entering a three-dimensional space bounded on the bottom and sides by the food cup and back of the shallow recess from which the pellet delivery chute extended. The top of this space was defined as the top of the flat face at the end of the pellet delivery chute. For animals that contributed only behavioral data, automatically registered beam breaks were used to calculate metrics. For analyses in which behavior was compared using both methods, "scoring method" was used as a factor in analyses and was found to not influence the results. 
Pavlovian conditioned approach (PCA) index was calculated to assess the degree of "sign-tracking" and "goal-tracking" exhibited by animals. The calculation of this metric was taken from Meyer et al. (2012). The index comprises three components, which are averaged together (for analysis of individual components, see Extended Data Fig. 1-1). The three metrics used in PCA index calculation are as follows: $\mathrm{CS}^{+}$over Goal approach preference: $\left[\left(\mathrm{CS}^{+}\right.\right.$approaches - Goalbox head entries)/ $\left(\mathrm{CS}^{+}\right.$approaches + Goalbox head entries)]; Probability of $\mathrm{CS}^{+}$over Goal approach: [ $\operatorname{Pr}\left(\mathrm{CS}^{+}\right.$Approach $)-\operatorname{Pr}($ Goal Approach $\left.)\right]$; Latency to approach: [mean ((latency to goal approach) - (latency to CS approach)) /10]. The difference in latency to approach was divided by $10 \mathrm{~s}$, the length of CS presentation, to place it on the same scale of -1 to 1 as the previous two metrics. Because of a computer error, CS Latency data failed to be recorded for 3 (of 59) animals on day 7. These animals were omitted from the PCA analysis.

Since the primary hypothesis being tested was that stress experience would alter learned approach behavior, some behavioral analyses compare "Early Training" with "Late Training." For most animals, Early Training included all conditioning trials on day 1 and Late Training included all conditioning trials on day 7 . There were four occurrences on day 1 and on day 7 in which an equipment malfunction prevented either proper behavioral or photometric recordings. In these cases, a subsequent conditioning day was used in the analyses. For the day 7 time point, this required 4 rats to be run in an additional conditioning session, day 8 , which was used to obtain Late Training data for analysis.

An ANOVA was used for all comparisons. In cases with multiple levels of a repeated-measure factor (i.e., analysis of CS approach over multiple days), sphericity assumptions were tested using Mauchly's test. Where this assumption was violated, the $p$ value of the affected test statistic was adjusted using the Hyun-Feldt estimated $\varepsilon$. Holm corrections were used to preserve family-wise error rate for all multiple comparisons. Statistical analyses were performed with R (https://www.r-project. org/).

\section{Fiber photometry}

A subset of animals that experienced the CVS procedure first received surgery for photometric recording to characterize the PL-NAcC activity patterns associated with the acquisition of conditioned approach.

Surgery. Animals to be used for photometry experiments were anesthetized under isoflurane (2.0\%-2.5\%) and head-fixed for stereotaxic implantation of an optic fiber targeting the PL and viral injection of GCaMP6f. Selective expression of the $\mathrm{Ca}^{2+}$ indicator GCaMP6f in NAcC-projecting PL neurons was accomplished using a dual viral approach. First, retrograde AAV2-CAG-Cre (University of North Carolina Vector Core) was injected into the core at two sites $\left(6^{\circ}\right.$; AP: $1.2 / 0.7 \mathrm{~mm}$; ML: $2.4 \mathrm{~mm}$; DV: $-5.0 \mathrm{~mm} ; 0.3 \mu \mathrm{l} / 3 \mathrm{~min} / \mathrm{site}$; titer $=8.1$ $\times 10^{12}$ molecules/ml). Next, AAV1-hSyn-FLEX-GCaMP6f-WPRE.SV40 (University of Pennsylvania Vector Core) was injected into the PL ( $8^{\circ}$; AP: $2.8 \mathrm{~mm}$; ML: $1.0 \mathrm{~mm}$; DV: $-4.0 \mathrm{~mm}$; $0.5 \mu \mathrm{l} / 5 \mathrm{~min} ; 6.5 \times 10^{12}$ molecules/ $\mathrm{ml}$ ) followed by optic fiber ( $5 \mathrm{~mm}$ length, $400 \mu \mathrm{m}$ core $/ 430 \mu \mathrm{m}$ outer diameter, 0.48 numerical aperture, flat tip; Doric) implantation ( $0^{\circ}$; AP: 2.8 $\mathrm{mm}$; ML: $0.6 \mathrm{~mm}$; DV: $-3.7 \mathrm{~mm}$ ) at the same site. Rats were treated with the anti-inflammatory drug, meloxicam ( $1 \%$ oral suspension), the day of surgery and for $4 \mathrm{~d}$ following surgery to reduce inflammation and postoperative pain.

Recording. Simultaneous recording of GCaMP6f fluorescence and background was accomplished using two separate wavelengths of light (465 and $405 \mathrm{~nm}$, respectively) provided by two single wavelength LEDs (Doric) controlled by an external dual channel driver (Doric), which itself was driven by an RZ5P processor (Tucker Davis Technologies). Both wavelengths were routed through a dichroic mirror (4-port fluorescence mini cube, Doric) and combined into a single $2 \mathrm{~m}$ jacketed patch cord ( $400 \mu \mathrm{m}$ core, 0.48 numerical aperture; Doric). This fiber was secured to the optic fiber implanted in the animal using a ceramic sleeve (Precision Fiber Products) and custom-made thumb screw clamp (University of Illinois, Chicago Machine Shop). This fiber carried both the excitation and emission fluorescence, which were separated by a dichroic mirror that delivered the GCaMP fluorescence to a Newport Visible Femtowatt photoreceiver (Doric; delivered by $600 \mu \mathrm{m}$ core/
$630 \mu \mathrm{m}$ outer diameter, 0.48 numerical aperture patch cord, Doric). Recordings occurred using commercially available software (Synapse; Tucker Davis Technologies) at $1017.2 \mathrm{~Hz}$ on each day of Pavlovian conditioning. Signal was recorded for at least $10 \mathrm{~min}$ before the beginning of each behavioral session to permit early signal decay. Behavior was videorecorded (10 frames/s) using a high-definition webcam (Logitech).

Data analysis. Data were extracted using scripts generously provided by the Lerner laboratory (Lerner et al., 2015) (https://github.com/ talialerner/Photometry-Analysis-Shared). A $40 \mathrm{~Hz}$ lowpass Butterworth filter was first applied to the $405 \mathrm{~nm}$ (isosbestic) signal. Then, both the 405 and $465 \mathrm{~nm}$ signals were downsampled by a factor of 10 from the original sampling rate. The processed isosbestic signal was fitted to the excitation signal using a linear fit to correct for signal decay. The GCaMP excitation signal was then normalized by subtracting the fitted isosbestic from it and dividing the difference by the fitted isosbestic, yielding the $\Delta \mathrm{F} / \mathrm{F}$.

The CS response was visualized by aligning the $\Delta \mathrm{F} / \mathrm{F}$ to $\mathrm{CS}$ events (10 s before CS onset and $20 \mathrm{~s}$ following). The signal during each trial was normalized relative to the baseline of that trial using a robust me$\operatorname{dian} Z$ score $[Z=(X-\tilde{x}) /(M A D)]$, where MAD $=\operatorname{Median}(X-\tilde{x})$ and $\tilde{\mathrm{x}}=$ the median $\Delta \mathrm{F} / \mathrm{F}$ during the $10 \mathrm{~s}$ pre-CS period for a given trial. Differences in activity around the presentation of the CS were calculated by examining different time epochs (10 s before CS presentation, $10 \mathrm{~s}$ during CS presentation, and $10 \mathrm{~s}$ after CS presentation). Aggregate activity for a given day (i.e., across 25 trials of a single type) was summarized as the area under the curve (trapezoidal estimation) of the average signal during these epochs.

Naturally occurring transient activity before a conditioning session was quantified by transient identification. Transients were counted as events in which activity exceeded 2.91 MADs as in Calipari et al. (2016). Transients were counted for the $5 \mathrm{~min}$ that immediately preceded the initiation of autoshaping training.

Investigator-scored time stamps of CS or Goal approach were used to compare activity patterns during individual CS and goal approach events. For each animal, the $z$-normalized $\Delta \mathrm{F} / \mathrm{F}$ signal during all approaches lasting at least $400 \mathrm{~ms}$ was extracted and averaged; $400 \mathrm{~ms}$ was selected following the qualitative assessment that the majority of approaches briefer than that threshold appeared incidental to an orienting response rather than an approach per se. On the last day of conditioning, most animals displayed both Goal-directed and CS-directed approach; however, 3 animals (2 Control, 1 Stress) made only CS approaches and thus contributed no Goal-directed approach signal data to the analysis. These animals were therefore excluded from the mixed ANOVA analyzing approach-related signal as missing data.

Statistical analyses were performed on CS type, stress condition, and training, and were conducted using mixed ANOVAs. Where appropriate, Welch's two-sample $t$ tests or the Mann-Whitney $U$ test (if the data did not meet the assumptions of parametric tests) were used to assess the effect of stress on certain measures. All statistics were performed in R.

\section{Experimental design}

All animals involved in photometry experiments recovered from surgery for $5 \mathrm{~d}$ before the initiation of CVS (or handling) procedures. From this time point, the experimental timeline was identical for both stressed and nonstressed animals. CVS was administered for $14 \mathrm{~d}$. Following the cessation of CVS, animals were left alone in their home cages with food and water available ad libitum for $7 \mathrm{~d}$ to allow for weight recovery in CVS animals. Food restriction to $95 \%$ body weight began $3 \mathrm{~d}$ before the initiation of autoshaping. Autoshaping training was conducted for at least $7 \mathrm{~d}$ for all animals.

\section{Results}

\section{Chronic stress impairs conditioned $\mathrm{CS}^{+}$approach}

Acquisition of conditioned cue approach was quantified as the probability of approach, calculated as the number of trials of a given type $\left(\mathrm{CS}^{+}\right.$or $\left.\mathrm{CS}^{-}\right)$in which the animal contacted the cue at least once divided by the total number of trials of that type. A 

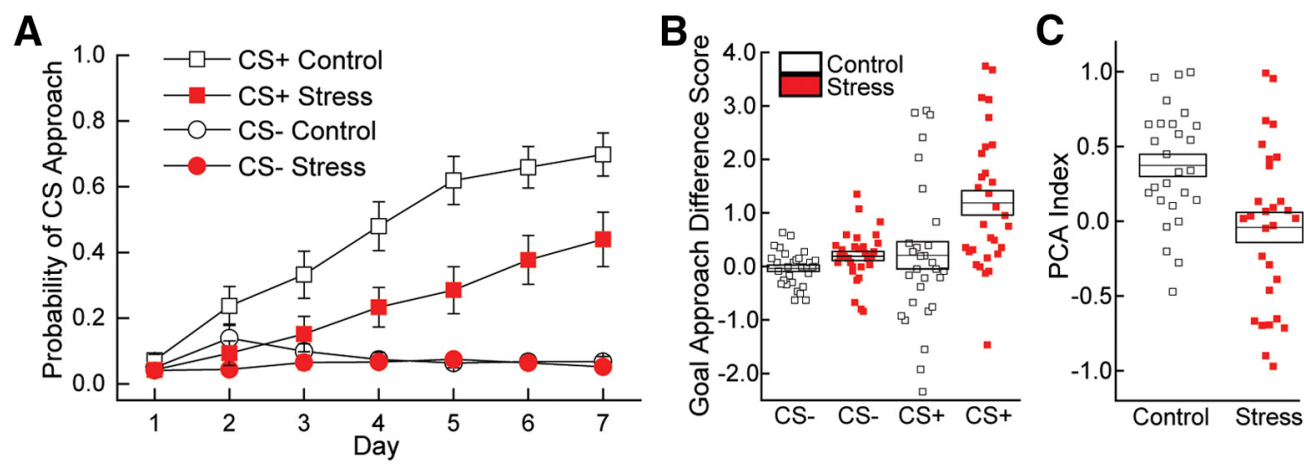

Figure 1. Chronic stress impairs conditioned approach directed at the $\mathrm{CS}^{+} . A$, Approach probability (mean \pm SEM) directed at the CS across daily training sessions. Chronic stress $(n=30)$ reduced $\mathrm{CS}^{+}$approach on day 7 compared with the Control condition $(n=29)\left(F_{(1,57)}=5.949, p=0.0358\right.$, Holm-corrected). The SS $^{-}$failed to promote approach in either condition. $\boldsymbol{B}$, On day 7, conditioned goal approach (mean \pm SEM) was quantified by calculating a difference score between goal approaches during the CS and goal approaches $10 \mathrm{~s}$ before the CS. Compared with the Control condition, chronic stress significantly increased goal approaches in response to the $\mathrm{CS}^{+}\left(F_{(1,57)}=9.493, p=0.00317\right)$. C, PCA index (mean \pm SEM) was calculated for all animals using $\mathrm{CS}^{+}$and goal approach behavior in the manner of Meyer et al. (2012) (for analysis of individual components, see Extended Data Figure 1-1). A positive score indicates $C S^{+}$-directed behavior, whereas a negative score indicates goal-directed behavior. This metric confirms the observation of a range of behavior in both groups, but a significant stress-induced change in approach behavior $\left(F_{(1,54)}=10.81, p=0.00178\right)$. Data from 3 control animals were not included in this analysis because of a computer error preventing the collection of $C S$ approach latency data (Control: $N=26 ;$ Stress: $N=30$ ).

2 (stress condition) $\times 2$ (CS type) $\times 7$ (day) mixed ANOVA was used to analyze the effect of conditioning, stress, and reward pairing on CS approach probability (Fig. 1A). There was a significant three-way interaction $\left(F_{(6,342)}=4.5127, p=0.002036\right.$; sphericity violated, Hyun-Feldt-corrected $p=0.004332)$. To interpret the three-way interaction, 2 (stress) $\times 7$ (day) mixed ANOVAs were run at both levels of CS. A significant interaction between stress condition and day was found for $\mathrm{CS}^{+}$approach $\left(F_{(6,342)}=2.9237\right.$, $p=0.008553$, sphericity violated, Hyun-Feldt-adjusted $p=0.03542$ ), but not $\mathrm{CS}^{-}$approach $\left(F_{(6,342)}=2.0488, p=0.05876\right.$, sphericity violated, adjusted $p=0.1100$ ). This interaction is explained by differences in the degree to which Stress and Control animals differed in their approach across days of training. Comparisons of approach on the first day of conditioning (day 1) and after conditioning (day $7)$ found there was no effect of stress on day 1 approach probability ( $p=0.197$, Holm-adjusted), but following $7 \mathrm{~d}$ of conditioning, Control animals (mean \pm SEM: $0.69 \pm 0.07$ ) were significantly more likely to approach the $\mathrm{CS}^{+}$than Stress animals $(0.44 \pm 0.08$; $p=0.0358)$. A separate analysis determined that stress also suppressed $\mathrm{CS}^{+}$interaction over the course of training in the subset of animals that contributed both behavioral and photometry data (Stress: $n=8$; Control: $n=13)\left(F_{(1,45)}=5.3576, p=0.0253\right]$, so these groups (behavior only and behavior + photometry) were combined for subsequent analyses.

\section{Chronic stress enhances conditioned goal approach}

Conditioned goal approach was also examined. Head entries into the area of the food cup were counted during both the $10 \mathrm{~s}$ CS presentation period and the preceding $10 \mathrm{~s}$ for each trial. The goal approach difference score for a given trial was calculated by subtracting the number of pre-CS head entries from head entries within the CS period during that trial. The count for all trials on the last day of conditioning was then averaged across all trials of the same CS type. A 2 (stress condition) $\times 2$ (CS type) mixed ANOVA found a significant interaction between stress condition and CS type on relative head entries $\left(F_{(1,57)}=6.1811, p=0.01586\right.$; Fig. $1 B)$. Animals in the stress condition made more relative head entries than Control animals during $\mathrm{CS}^{+}$presentation $\left(F_{(1,57)}=\right.$ 9.493, $p=0.00317$, partial $\left.\eta^{2}=0.143\right)$ and $\mathrm{CS}^{-}$presentation $\left(F_{(1,57)}=\right.$ 4.698, $p=0.0344$ partial $\left.\eta^{2}=0.076\right)$, but this effect was much larger on $\mathrm{CS}^{+}$trials (Stress: $1.29 \pm 0.24$; Control: $0.22 \pm 0.26$ ) than $\mathrm{CS}^{-}$trials (Stress: $0.20 \pm 0.09$; Control: $-0.03 \pm 0.06$ ).
Individual differences in the tendency to engage in conditioned $\mathrm{CS}^{+}$or Goal approach behavior were examined by calculating a composite PCA index in the manner of Meyer et al. (2012). This score was calculated as the average of three metrics: $\mathrm{CS}^{+}$over Goal approach preference; Probability of $\mathrm{CS}^{+}$over Goal approach; and Latency to approach. This index falls on a scale between -1 (indicating exclusively Goal approach) and 1 (indicating exclusively $\mathrm{CS}^{+}$approach). A one-way ANOVA compared the PCA indices of Stress and Control animals on the last day of conditioning. There was a significant effect of stress experience $\left(F_{(1,54)}=10.81, p=0.00178\right)$, such that Control animals as a group displayed more $\mathrm{CS}^{+}$approach behavior $(0.37 \pm 0.07)$, whereas Stress animals engaged in more goal approach behavior $(-0.04 \pm 0.10$; Fig. $1 C)$.

\section{Photometry}

Chronic stress does not alter naturally occurring, non-taskrelated, PL-NAcC activity

Selective expression of GCaMP6(f) in NAcC-projecting PL neurons combined with optic-fiber implantation in the PL was used to monitor PL-NAcC activity during autoshaping conditioning (Fig. 2) in a subset of animals. Signal was recorded for the duration of the behavioral session and for at least $5 \mathrm{~min}$ before the first CS presentation on each conditioning day. Non-taskrelated, naturally occurring coordinated neural activity was examined by identifying and comparing transient activity in Control and Stress conditions. A transient was defined as any period in which the $\Delta \mathrm{F} / \mathrm{F}$ exceeded 2.91 median absolute deviations (Fig. $3 A, B)$. A $2 \times 2$ mixed ANOVA was conducted to compare the effect of conditioning on transient activity during this baseline period in both Stress and Control animals. Neither main effects (stress condition: $F_{(1,19)}=0.0849, p=0.7739$; day: $F_{(1,19)}=$ $0.9465, p=0.3428)$ nor an interaction $\left(F_{(1,19)}=1.6156\right.$, $p=0.2190$ ) was found (Fig. $3 C$ ).

\section{Chronic stress attenuates $\mathrm{CS}^{+}$encoding in PL-NAcC neurons}

Task-related activity on each conditioning trial was examined across days for each animal. Representative color plots of activity illustrate differences in the development of activity related to the $\mathrm{CS}$ and reward in Control and Stress animals (Fig. 4A). This difference was analyzed at the beginning (Early Training) and last day of conditioning (Late Training) (Fig. 4B) to test the 
hypothesis that stress disrupts conditioned cue encoding. A 2 (Stress condition) $\times 2$ (Early vs Late Training) $\times 2$ (CS type) mixed ANOVA analyzed the area under the curve of the signal during the $10 \mathrm{~s}$ CS presentation (Fig. 4C). This analysis found a significant interaction between Stress experience and Training $\left(F_{(1,19)}=5.8014, p=0.02633\right)$ and a significant main effect of CS type $\left(F_{(1,19)}=\right.$ $8.2782, p=0.00965)$. To test the hypothesis that stress experience would interfere with the acquisition of $\mathrm{CS}^{+}$encoding, the planned comparison did not include the $\mathrm{CS}^{-}$response. One-way (Early vs Late Training) ANOVAs at each level of Stress found a significant effect of Training on signal magnitude for Control animals $\left(F_{(1,12)}=5.3627, \quad p=0.0391 ; \quad\right.$ Early Training: $11.16 \pm 2.24$; Late Training: $19.53 \pm 4.47)$ but not Stress animals $\left(F_{(1,7)}=0.1515, p=0.709\right.$; Early Training: $4.47 \pm 1.19$; Late Training: $5.32 \pm 2.72$ ).

Similar analyses examined the $10 \mathrm{~s}$ period following the termination of the CS. This period coincides with reward delivery on $\mathrm{CS}^{+}$trials. For this period, a significant interaction was found between Stress experience and CS type $\left(F_{(1,19)}=\right.$ 4.7677, $p=0.0417)$ as was a significant main effect of Training $\left(F_{(1,19)}=9.1781\right.$, $p=0.006895$ ), such that autoshaping increased pathway activity in the period following CS termination across groups. The twoway Stress $\times$ CS type interaction was interpreted by collapsing across Training (by averaging the epoch-magnitudes across early and late training for each animal) and performing separate oneway ANOVAs on CS type for Control and Stress animals. Only Control animals showed a significant increase in activity during the post- $\mathrm{CS}^{+}$period relative to the post- $\mathrm{CS}^{-}$period $\left(F_{(1,12)}=\right.$ $6.9632, p=0.0216$; post-CS ${ }^{+}: 17.81 \pm 3.19$; post-CS $: 7.50 \pm$ $1.65)$. Stress animals did not display a difference in their encoding of the post-CS ${ }^{+}$and post-CS ${ }^{-}$periods $\left(F_{(1,7)}=1.1305\right.$, $p=0.322$; post-CS ${ }^{+}: 7.99 \pm 2.88$; post-CS $\left.: 4.88 \pm 1.88\right)$. Similarly, a direct comparison between the late training Post-CS ${ }^{+}$ signal in Control and Stress animals revealed that Control animals displayed greater signal during reward delivery than did Stress animals (Welch $T_{(18.717)}=2.276, p=0.03479$ ). Together, these results indicate that, unlike in Control animals, pathway activity in animals with Stress experience did not discriminate between reward delivery and the absence thereof. Stress and Control animals did not differ in their latency to retrieve the pellet from the food cup following $\mathrm{CS}^{+}$ retraction $(U=93, p>0.05)$. Thus, the difference in signal cannot be attributed to a failure or delay on the part of Stress animals in retrieving and consuming the reward. Furthermore, the magnitude of reward encoding did not correlate with individual differences in approach behavior (as measured by PCA index $)$ in either Stress $(r=0.102, p=0.811)$ or Control $(r=-0.404, p=0.171)$ animals. Therefore, the difference between Stress and Control animals in their encoding the post- $\mathrm{CS}^{+}$period relative the post- $\mathrm{CS}^{-}$period cannot be attributed to the greater propensity of stress animals to engage in goal-tracking.
B

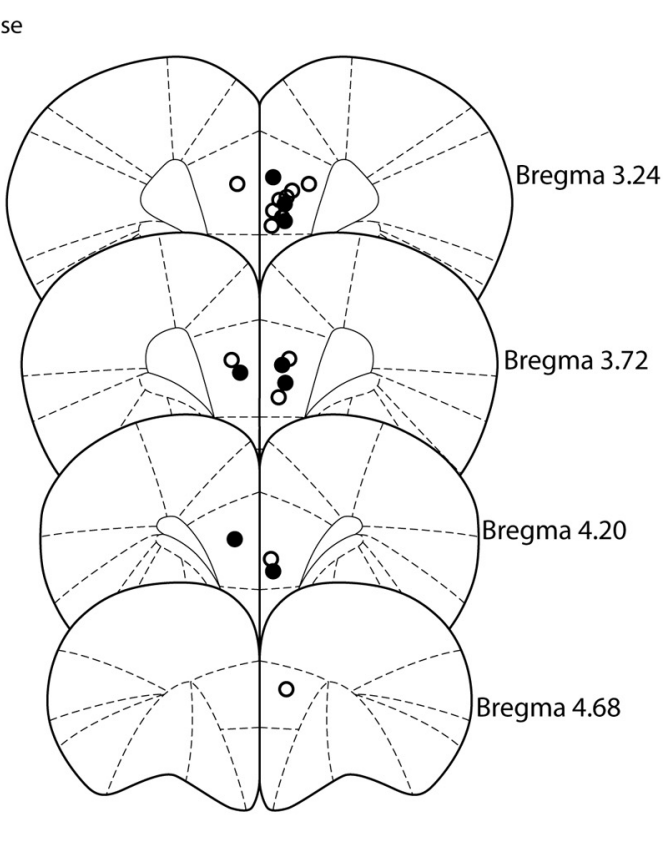

Figure 2. Technical approach for fiber-photometric monitoring of PL-NAcC activity. $\boldsymbol{A}$, Viral strategy for selective expression histologically verified fiber tips that terminated in a region of the PL in which GCaMP6(f) expression was also verified. Open PL-NAcC activity does not predict the direction of conditioned approach

The possibility that quantitatively different PL-NAcC activity patterns could be associated with different types of approach behavior $\left(\mathrm{CS}^{+}\right.$vs Goal) was also examined on the last day of conditioning (Fig. 5A). An approach was defined as the animal contacting either the CS or food cup. Time stamps of both initiation and cessation of approach were marked, and the average signal during these types of approach was calculated. A 2 (Stress vs Control $) \times 2$ (CS vs Goal) ANOVA found a significant main effect of Stress experience $\left(F_{(1,16)}=8.617, p=0.009699\right)$, but no effect of Approach type $\left(F_{(1,16)}=2.16, p=0.161\right)$ nor an interaction $\left(F_{(1,16)}=0.0032, p=0.955\right.$; Fig. $\left.5 B\right)$. To assess the possibility that variability in signal magnitude related to individual behavioral variability, Pearson correlations were performed between average signal during $\mathrm{CS}^{+}$approach and $\mathrm{CS}^{+}$approach probability within both Stress and Control groups (Fig. 5C). Neither Stress $(r=0.034, p=0.943)$ nor Control $(r=-0.094, p=0.784)$ groups showed a significant correlation between signal and preferred direction of approach behavior.

To further examine the relationship between activity and approach behavior, a $2 \times 2$ between-subjects ANOVA was used to analyze the magnitude of $\mathrm{CS}^{+}$encoding as a factor of stress condition and the PCA index (grouped by those $>0.5$ and those $<0.5$ ). A significant main effect of stress was found on CS signal $\left(F_{(1,17)}=4.587, p=0.047\right)$, but the interaction between stress and PCA grouping was not significant $\left(F_{(1,17)}=0.029, p=0.866\right)$. The observation of a significant main effect of stress on CS signal with the absence of a significant stress condition $\times$ PCA condition interaction is consistent with the results of the correlation analyses. Together, they support the interpretation that the reduced PLCore activity in response to the $\mathrm{CS}^{+}$is a result of stress exposure rather than a physiological correlate of goal-tracking behavior. 
A

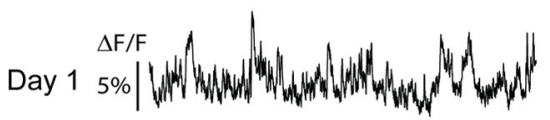
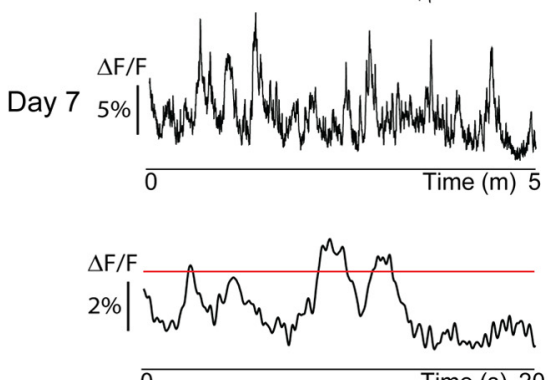

B

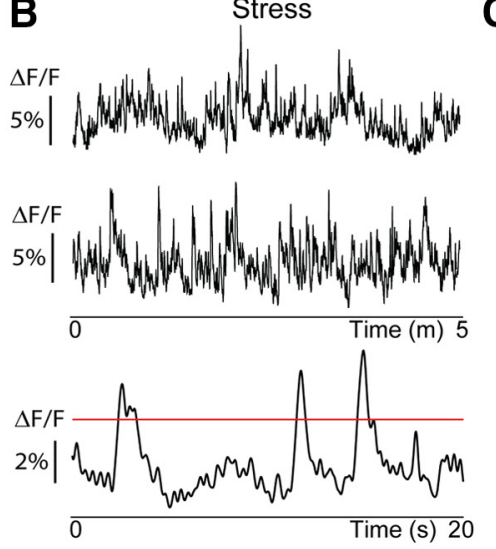

C

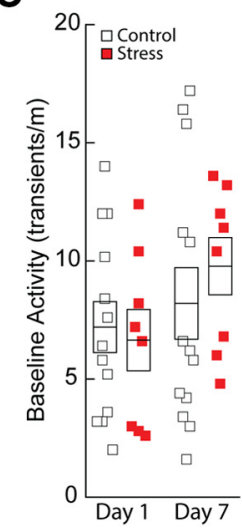

Figure 3. Chronic stress does not alter non-task-related transient activity. Naturally occurring, non-task-related activity from a single Control $(\boldsymbol{A})$ and Stress $(\boldsymbol{B})$ animal. Each recording occurred $5 \mathrm{~min}$ before the onset of the autoshaping session on the first day (top) and last day (middle) of conditioning. Recordings were used to identify and count transients (activity peaks $>2.91$ median absolute deviations [MAD]). Bottom, A $20 \mathrm{~s}$ segment of an above trace with the MAD illustrated as a red horizontal line. $C$, Non-task-related activity was quantified for all animals in Stress $(n=8)$ and Control $(n=13)$ conditions. There was neither an effect of conditioning $\left(F_{(1,19)}=0.9465, p=0.3428\right)$ nor an effect of stress $\left(F_{(1,19)}=0.0849, p=0.7739\right)$ on transient frequency.

A
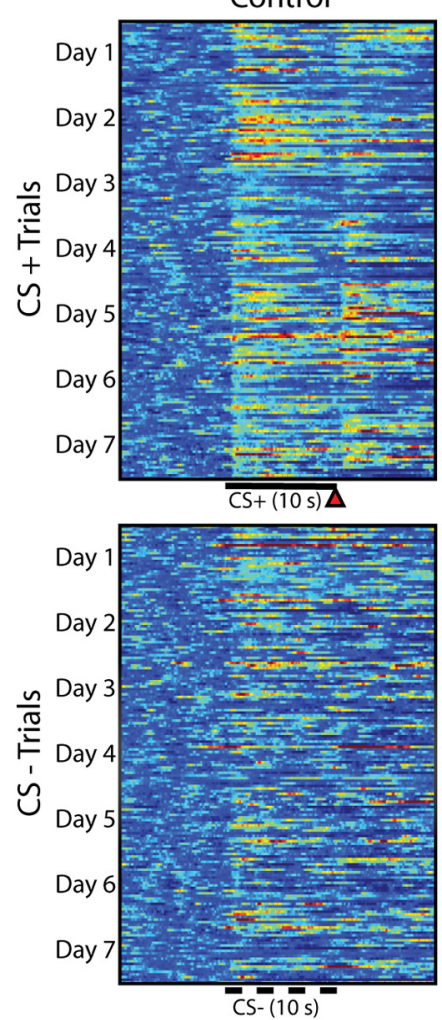

Stress

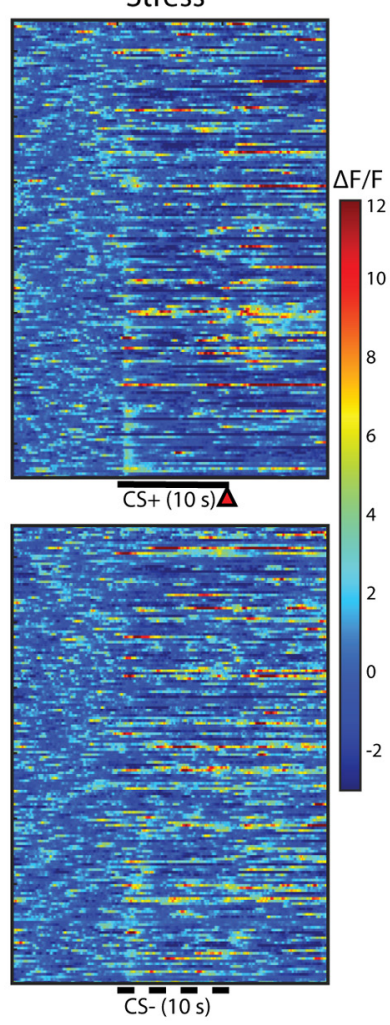

B

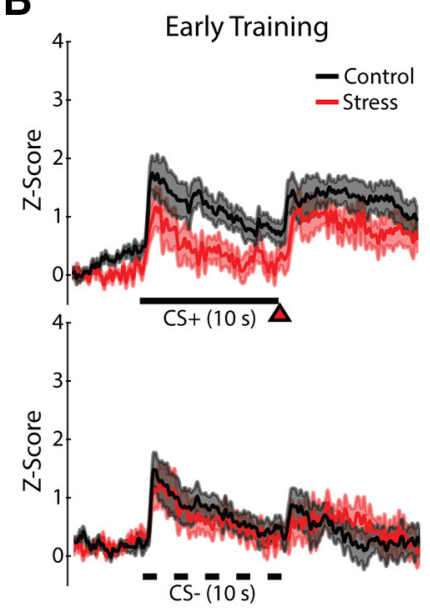

C ${ }_{60}$ CS+ Epoch Post CS+

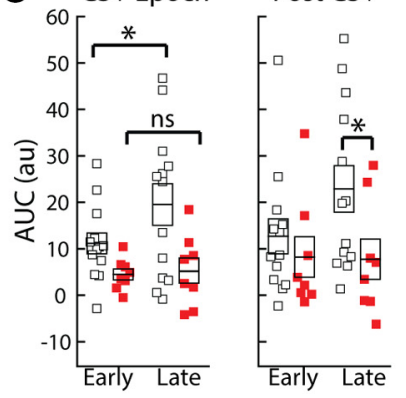

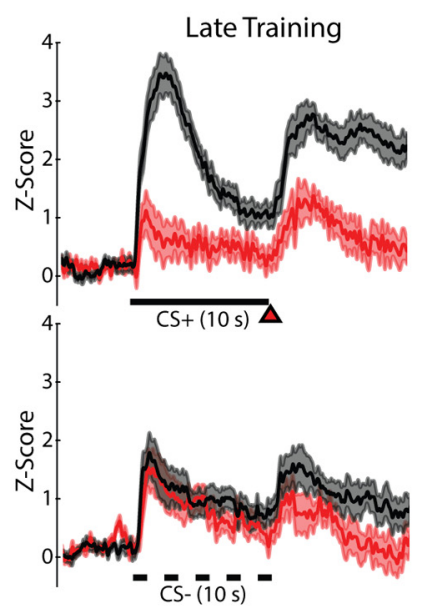

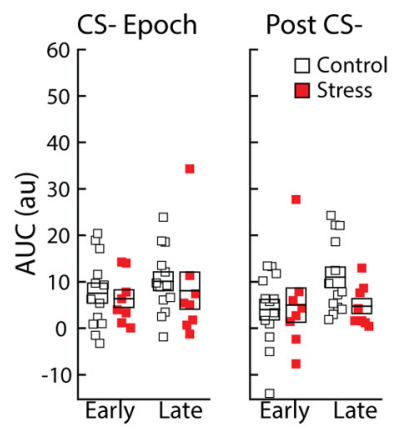

Figure 4. Chronic stress reduces $\mathrm{CS}^{+}$evoked PL-NAcC Activity. A, Representative fiber photometric monitoring of trial $\times$trial $\mathrm{CS}^{+}$and $\mathrm{CS}^{-}$encoding in PL-NAcC neurons over $7 \mathrm{~d}$ of conditioning in a Control and Stress animal. Thirty second traces of $\triangle \mathrm{F} / \mathrm{F}$ (represented in pseudo-color) are aligned to $175 \mathrm{CS}^{+}$(solid line) and $\mathrm{CS}^{-}$(dashed line) trials for representative Control and Stress animals. Red triangle represents reward delivery. B, Mean ( \pm SEM in shaded area) PL-NACC activity during the beginning (Early Training) and last day (Late Training) of conditioning during $\mathrm{CS}^{+}$(solid line) and $\mathrm{CS}^{-}$(dashed line) trials. Red triangle represents reward delivery. C, Activity (mean \pm SEM) during the $10 \mathrm{~s}$ CS period and $10 \mathrm{~s}$ post-CS period was quantified as area under the curve (AUC) for each animal in each condition. In Control animals $(n=13)$, conditioning significantly increased pathway activity during $C S^{+}\left(F_{(1,12)}=5.3627, p=0.0391\right)$, but not $\mathrm{CS}^{-}$, trials. Stress $(n=8)$ prevented this effect. Control animals also showed a significant increase in activity during the post- $\mathrm{CS}^{+}$period following conditioning, but not during the post-CS ${ }^{-}$pe$\operatorname{riod}\left(F_{(1,12)}=6.9632, p=0.0216\right)$. *indicates significance, $p<0.05$. ns, not significant. Animals in the stress condition did not significantly alter pathway activity following training.

\section{Discussion}

The results presented in this report demonstrate that prior chronic stress exposure both disrupts the encoding of a cue that promotes approach behavior and alters the nature of conditioned approach. Pavlovian autoshaping is a behavioral design in which a physical cue $\left(\mathrm{CS}^{+}\right)$predicts the delivery of a reward, usually food. Animals trained in this design develop a conditioned approach during cue presentation directed toward either the cue 

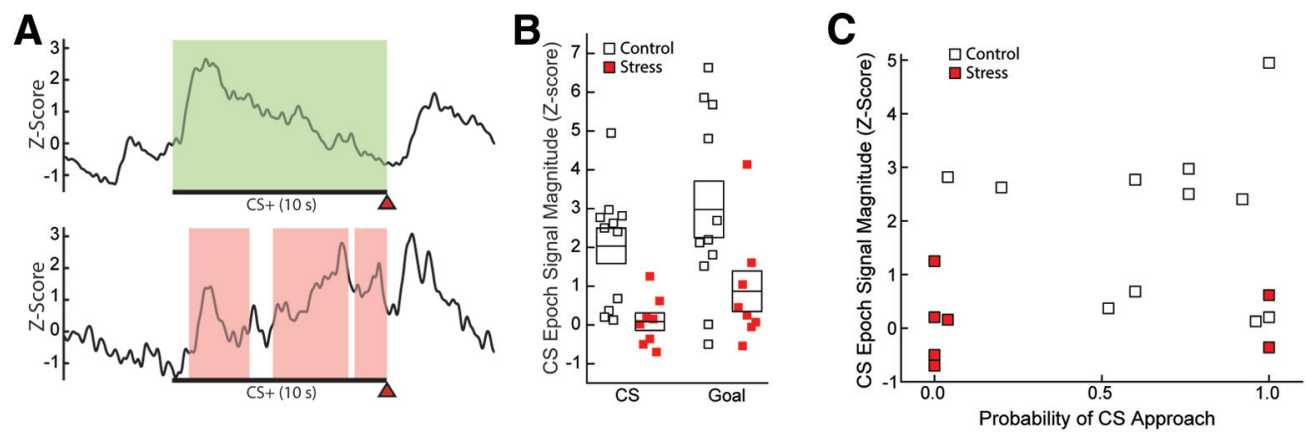

Figure 5. PL-NACC activity is present during both $\mathrm{CS}^{+}$and Goal approach behavior. $A$, Representative traces from individual trials in which Control animals displayed either a single $\mathrm{CS}^{+}$ approach (top, behavior for the duration of green overlay) or 3 separate Goal approaches (bottom, behavior for the duration of each red overlay). Red triangle represents reward delivery. $\boldsymbol{B}$, PL-NAcC Activity (mean \pm SEM) during different types of approach for Stress $(n=7)$ and Control $(n=11)$ animals that displayed both types of approach. Activity during CS versus Goal approaches did not differ $\left(F_{(1,16)}=2.16, p=0.161\right)$. Stress induced a general reduction in activity regardless of approach type $\left(F_{(1,16)}=8.617, p=0.0097\right)$. $C$, Individual differences in approach behavior and PL-NACC activity in response to the $\mathrm{CS}^{+}$. Variability in the average signal during CS-prompted approaches is not explained by individual variation in the probability of exhibiting $\mathrm{CS}^{+}$approach (Stress: $r=0.034, p=0.943$; Control: $r=-0.094, p=0.784$ ).

itself or the site of reward delivery. These two types of conditioned responses are thought to reflect different kinds of cue learning: reward port-approaching animals, referred to as goal trackers, appear to assign only predictive value to the $\mathrm{CS}^{+}$, while for cue-approaching animals, referred to as sign trackers, the $\mathrm{CS}^{+}$appears to also take on incentive value (Robinson and Flagel, 2009). Most animals in this report displayed a mixed approach. A given animal's relative likelihood to approach the cue over the food cup can be described using a compound metric, the PCA index (Meyer et al., 2012), which averages the relative preference for $\mathrm{CS}^{+}$over goal approach, the relative likelihood of $\mathrm{CS}^{+}$versus goal approach, and the relative latency of $\mathrm{CS}^{+}$to goal approach. Using this metric, most nonstressed animals were found to develop a preference for the $\mathrm{CS}^{+}$(as indicated by PCA index values $>0$ ). However, experience with CVS not only reduced the tendency of animals to develop sign-tracking, but also significantly elevated their propensity to goal-track. Because both types of responses to the cue are learned, it is clear that stress did not impair the ability to either learn the predictive nature of a stimulus or develop and express a conditioned response to that stimulus. Instead, animals that are most susceptible to stress appear to have a specific deficit in incentive learning and are capable of ascribing only predictive value to the reward-paired cue. This interpretation is consistent with prior research reporting intact associative learning, but disrupted cue-incentivized responding in stress-exposed animals (Morgado et al., 2012).

Although chronic stress procedures have long been used to induce dysfunction in reward processing (Willner, 2017), the precise nature of that dysfunction has been the subject of recent debate. It has been proposed that stress-induced disruptions in reward processing are better aligned with amotivation or avolition than anhedonia, the inability to experience pleasure (Olney et al., 2018; Rizvi et al., 2018). While the present study was not designed to determine whether stress selectively disrupts hedonic processing, our laboratory has not observed stress-induced hedonic processing disruptions measured with the taste reactivity procedure (M.G.S., unpublished observations). Additionally, it was noted that all animals consumed the sucrose reward on all trials in the current study. Therefore, these data are consistent with the view that stress disrupts reward processing by interfering with the ability of rewarding stimuli to properly motivate behavior. In this case, rewarding stimuli failed to support the acquired incentive value of a CS. More work will be needed to identify the specific nature of the incentive deficit, and whether it arises at the level of perception, representation, or transfer to cues.
The effects of stress on sign-tracking appear to depend on the nature or timing of the stress. A similar prolonged stress procedure produced behavioral effects similar to those observed herein (Fitzpatrick et al., 2019), whereas social isolation during adolescence was shown to increase signtracking in adulthood (Beckmann and Bardo, 2012), which has been associated with the development of sensitization thought to promote compulsive drug seeking (Berridge and Robinson, 1995). Thus, neither sign-tracking nor goal-tracking should be interpreted as evidence of pathology per se. Instead, these behaviors are an enormously valuable tool for understanding how experience modifies specific neural pathways that regulate motivated behavior, as some of these modifications may align with specific symptoms of psychopathology.

The $\mathrm{mPFC}$ has a particular role in learning reward contingencies (Balleine and Dickinson, 1998), and the PL subregion (in particular via its efferents to the NAcC) is necessary for cuedirected motivated behavior (McFarland et al., 2003; Otis et al., 2017). Glutamate in this pathway tracks $\mathrm{CS}^{+}$presentation in sign-tracking animals (Batten et al., 2018). The present study used fiber photometric recording of PL-NAcC projection neurons to monitor activity in this pathway during autoshaping training in stressed and nonstressed animals. Activity in this pathway emerged as the $\mathrm{CS}^{+}$came to predict reward delivery only in nonstressed animals. Nonetheless, PL-NAcC neurons did not become quiescent following stress; transient analysis of presession baseline activity found no difference in the rate of naturally occurring, non-task-related, activity. This finding recalls the context-specific, rather than resting-state, deficits observed in corticolimbic connectivity in people who suffer from major depressive disorder (Young et al., 2016). The lack of a difference in non-task-related activity suggests that, following stress, neurons in this pathway may be insensitive to drive from other inputs, these inputs may themselves be compromised, or both.

In this autoshaping task, the activity of PL-NAcC neurons did not appear to predict the likelihood that a given approach was directed at the $\mathrm{CS}^{+}$or the goal. As stated above, most animals in this study engaged in a mixed approach, exhibiting both $\mathrm{CS}^{+}$ approaches and goal approaches while also exhibiting a tendency to engage in one type of approach at the expense of the other. These individual differences were closely examined to determine whether either type of approach was better aligned with PL$\mathrm{NAcC}$ activity and no predictive relationship was found. It would be interesting to compare these results with other study designs that engender more $\mathrm{CS}^{+}$approach behavior or goal-approach 
behavior. However, Figure $5 \mathrm{~A}$ illustrates the remarkable similarity in signal that was typically observed when individual trials were closely examined in which animals exhibited a $\mathrm{CS}^{+}$ approach or a goal approach. Increased PFC-NAcC activity was typically associated with both. This may reflect the complex role of the PFC in regulating conditioned approach behavior. PL neural activity has been proposed to provide top-down inhibition of autoshaping behavior that helps the animal to maintain focus on the primary reward rather than the cue (Paolone et al., 2013; Campus et al., 2016). However, autoshaping behavior is associated with glutamate release in the mPFC, and lesions of the mPFC can reduce autoshaping behavior (Batten et al., 2018; Serrano-Barroso et al., 2019). While the PL-NAc pathway was significantly less active during this task in stressed animals, they continued to goal-track and consume the reward, suggesting that PL-NAcC activity is not necessary for those behaviors. The similar activity patterns in nonstressed sign- and goal-tracking animals suggest that PL-NAcC activity is not sufficient to cause the acquisition or expression of approach behavior directed toward the cue. It is likely, then, that PL-NAcC activity contributes to, but is not required for, this behavior. This complexity may reflect the function of other PFC projection targets, such as the paraventricular thalamus (PVT), which act to inhibit conditioned cue approach behavior. Increased activity of the PL-PVT pathway interferes with cue-directed behavior, whereas disruption of this pathway promotes attending to the cue in a similar design (Campus et al., 2019). It is possible that PL-PVT activity competes with PL-NAcC activity to direct behavior toward the goal or cue, respectively. However, when PL-NAcC activity is compromised, as after chronic stress, the balance for behavioral control is shifted.

Alternatively, these findings may indicate that stress induces a fundamental change in the nature by which animals learn and engage in behavior. Chronic stress induces atrophy in mPFC neurons (Radley et al., 2006; Dias-Ferreira et al., 2009) while simultaneously leading to hypertrophy of sensorimotor cortices (Dias-Ferreira et al., 2009). Consistent with other Pavlovian approach designs, nonstressed rats learned to associate the $\mathrm{CS}^{+}$with reward delivery and express a conditioned response via a circuit that includes PL-NAcC projections (Otis et al., 2017). That stressed animals continued to express a conditioned response in the absence of PLNAcC activity may indicate that learning in these animals relied on separate neural circuits. Additional research using intersectional approaches can test these provocative hypotheses and probe the mechanism of the altered activity patterns characterized in this report.

This study contributes to an emerging understanding of both how a stressful experience interferes with the acquisition of learned approach and how stress changes brain circuits involved in approach behavior. Using an intersectional approach, this report characterizes the involvement of the PL-NAcC pathway in the typical acquisition of learned approach directed at both the incentivized cue and the goal. Further, the data characterize the reduction in cue-directed behavior that accompanies stress and is associated with severely impaired cortical activity. These findings support the interpretation that stress disrupts reward processing by altering the incentive value of cues. Perhaps most interesting is the possibility that the emergent behavior may be rooted in altered circuitry available for learning. Future work may characterize the mechanisms by which typically used brain circuits are dysregulated by stress, and how the roles of these circuits are transferred to other areas.

\section{References}

Balleine BW, Dickinson A (1998) Goal-directed instrumental action: contingency and incentive learning and their cortical substrates. Neuropharmacology 37:407-419.

Batten SR, Pomerleau F, Quintero J, Gerhardt GA, Beckman JJ (2018) The role of glutamate signaling in incentive salience: second-by-second glutamate recordings in awake Sprague-Dawley rats. J Neurochem 145:276286.

Beckmann JS, Bardo MT (2012) Environmental enrichment reduces attribution of incentive salience to a food-associated stimulus. Behav Brain Res 226:331-334.

Berridge KC, Robinson TE (1995) The mind of an addicted brain: neural sensitization of wanting versus liking. Curr Dir Psychol Sci 4:71-76.

Berridge KC, Robinson TE, Aldridge JW (2009) Dissecting components of reward: 'liking,' 'wanting,' and learning. Curr Opin Pharmacol 9:65-73.

Calipari ES, Bagot RC, Purushothaman I, Davidson TJ, Yorgason JT, Peña CJ, Walker DM, Pirpinias ST, Guise KG, Ramakrishnan C, Deisseroth K, Nestler EJ (2016) In vivo imaging identifies temporal signature of D1 and D2 medium spiny neurons in cocaine reward. Proc Natl Acad Sci USA 113:2726-2731.

Campus P, Accoto A, Maiolati M, Latagliata C, Orsini C (2016) Role of prefrontal 5-HT in the strain-dependent variation in sign-tracking behavior of C57BL/6 and DBA/2 mice. Psychopharmacology (Berl) 233:11571169.

Campus P, Covelo IR, Kim Y, Parsegian A, Kuhn BN, Lopez SA, Neumaier JF, Ferguson SM, Solberg Woods LC, Sarter M, Flagel SB (2019) The paraventricular thalamus is a critical mediator of top-down control of cue-motivated behavior in rats. eLife 8:e49041.

Davey GC, Cleland GG (1982) Topography of signal-centered behavior in the rat: effects of deprivation state and reinforcer type. J Exp Anal Behav 38:291-304.

Dias-Ferreira E, Sousa JC, Melo I, Morgado P, Mesquita AR, Cerqueira JJ, Costa RM, Sousa N (2009) Chronic stress causes frontostriatial reorganization and affects decision-making. Science 325:621-625.

Drysdale AT, Grosenick L, Downar J, Dunlop K, Mansouri F, Meng Y, Fetcho RN, Zebley B, Oathes DJ, Etkin A, Schatzberg AF, Sudheimer K, Keller J, Mayberg HS, Gunning FM, Alexopoulos GS, Fox MD, PascualLeone A, Voss HU, Casey BJ, et al. (2017) Resting-state connectivity biomarkers define neurophysiological subtypes of depression. Nat Med 23:28-38.

Fitzpatrick CJ, Jagannathan L, Lowenstein ED, Robinson TE, Becker JB, Morrow JD (2019) Single prolonged stress decreases sign-tracking and cue-induced reinstatement of cocaine-seeking. Behav Brain Res 359:799806.

Insel TR, Cuthbert BN (2015) Brain disorders? Precisely. Science 348:499500 .

Lerner TN, Shilyansky C, Davidson TJ, Evans KE, Beier KT, Zalocusky KA, Crow AK, Malenka RC, Luo L, Tomer R, Deisseroth K (2015) Intactbrain analyses reveal distinct information carried by SNc dopamine subcircuits. Cell 162:635-647.

McFarland K, Lapish CC, Kalivas PW (2003) Prefrontal glutamate release into the core of the nucleus accumbens mediates cocaine-induced reinstatement of drug-seeking behavior. J Neurosci 23:3531-3537.

Meyer PJ, Lovic V, Saunders BT, Yager LM, Flagel SB, Morrow JD, Robinson TE (2012) Quantifying individual variation in the propensity to attribute incentive salience to reward cues. PLoS One 7:e38987.

Morgado P, Silva M, Sousa N, Cerqueira JJ (2012) Stress transiently affects pavlovian-to-instrumental transfer. Front Neurosci 6:93-96.

Olney JJ, Warlow SM, Naffziger EE, Berridge KC (2018) Current perspectives on incentive salience and applications to clinical disorders. Curr Opin Behav Sci 22:59-69.

Otis JM, Namboodiri VM, Matan AM, Voets ES, Mohorn EP, Kosyk O, McHenry JA, Robinson JE, Resendez SL, Rossi MA, Stuber GD (2017) Prefrontal cortex output circuits guide reward seeking through divergent cue encoding. Nature 543:103-107.

Paolone G, Angelakos CC, Meyer PJ, Robinson TE, Sarter M (2013) Cholinergic control over attention in rats prone to attribute incentive salience to reward cues. J Neurosci 33:8321-8335.

Price RB, Duman R (2020) Neuroplasticity in cognitive and psychological mechanisms of depression: an integrative model. Mol Psychiatry 25:530543. 
Radley JJ, Rocher AB, Miller M, Janssen WG, Liston C, Hof PR, McEwen BS, Morrison JH (2006) Repeated stress induces dendritic spine loss in the rat medial prefrontal cortex. Cereb Cortex 16:313-320.

Ribot T (1896) La Psychologie des Sentiments. Ancient Philosophy 7:287.

Rizvi SJ, Lambert C, Kennedy S (2018) Presentation and neurobiology of anhedonia in mood disorders: commonalities and distinctions. Curr Psychiatry Rep 20:6-9.

Robinson TE, Flagel SB (2009) Dissociating the predictive and incentive motivational properties of reward-related cues through the study of individual differences. Biol Psychiatry 65:869-873.

Serrano-Barroso A, Vargas JP, Diaz E, O’Donnell P, López JC (2019) Sign and goal tracker rats process differently the incentive salience of a conditioned stimulus. PLoS One 14:e0223109.
Thomsen KR, Whybrow PC, Kringelbach ML (2015) Reconceptualizing anhedonia: novel perspectives on balancing the pleasure networks in the human brain. Front Behav Neurosci 9:49.

Vialou V, Bagot RC, Cahill ME, Ferguson D, Robison AJ, Dietz DM, Fallon B, Mazei-Robison M, Ku SM, Harrigan E, Winstanley CA, Joshi T, Feng J, Berton O, Nestler EJ (2014) Prefrontal cortical circuit for depressionand anxiety-related behaviors mediated by cholecystokinin: role of FosB. J Neurosci 34:3878-3887.

Willner P (2017) The chronic mild stress (CMS) model of depression: history, evaluation and usage. Neurobiol Stress 6:78-93.

Young CB, Chen T, Nusslock R, Keller J, Schatzberg AF, Menon V (2016) Anhedonia and general distress show dissociable ventromedial prefrontal cortex connectivity in major depressive disorder. Transl Psychiatry 6:e810. 